PROCEEDINGS OF THE

AMERICAN MATHEMATICAL SOCIETY

Volume 131, Number 12, Pages 3913-3917

S 0002-9939(03)06969-7

Article electronically published on March 25, 2003

\title{
AN EXAMPLE OF A $C$-MINIMAL GROUP WHICH IS NOT ABELIAN-BY-FINITE
}

\author{
PATRICK SIMONETTA \\ (Communicated by Carl G. Jockusch, Jr.)
}

\begin{abstract}
In 1996 D. Macpherson and C. Steinhorn introduced $C$-minimality as an analogue, for valued fields and some groups with a definable chain of normal subgroups with trivial intersection, of the notion of o-minimality. One of the open questions of that paper was the existence of a non abelian-by-finite $C$-minimal group. We give here the first example of such a group.
\end{abstract}

\section{INTRODUCTION}

The notion of o-minimality has undergone a very important development in recent years and has found many applications, for example in the study of expansions of real closed fields by analytic functions. Recall that o-minimal structures are totally ordered structures in which the parameter-definable subsets are finite unions of intervals with endpoints in the structure. More recently D. Macpherson and C. Steinhorn introduced $C$-minimality in [5] as a variant of the notion of o-minimality. In a $C$-minimal structure, a ternary relation, with some specific properties, the $C$-relation plays the role analogous to the order in an o-minimal structure: any parameter-definable subset is quantifier-free definable with formulae using just the $C$-relation and equality. Such relations arise naturally in valued groups and fields. Less developed than o-minimality for the moment, this notion has already led to some promising results (see [5] and [1]). It applies to expansions of algebraically closed valued fields ([4]), and may be expected to have a development in some ways analogous to o-minimality (see [1]). Some of the tools of stability can be developed in this context ([2], 3]). Notwithstanding, some basic questions remain: while, as in the o-minimal case, $C$-minimal fields are characterized, they are exactly the algebraically closed valued fields, $C$-minimal groups are far less understood than the o-minimal: we do not know which groups can be endowed with a $C$-minimal structure. There are many examples of abelian $C$-minimal groups (see [5], [7]) and it is easy to construct non-abelian $C$-minimal groups by adding a finite non-abelian group to an abelian $C$-minimal group as a direct summand. However, up to now, there have been no examples of non-abelian-by-finite $C$-minimal groups. In this paper we give such an example, the first one as far as I know, answering a

Received by the editors May 25, 2001 and, in revised form, July 25, 2002.

2000 Mathematics Subject Classification. Primary 03C60; Secondary $20 \mathrm{~F} 18$.

Key words and phrases. $C$-minimal groups, $C$-minimality, o-minimality, algebraically closed valued fields, nilpotent groups. 
question of D. Macpherson. While $C$-minimality is proved in general using a quantifier elimination result, our group is obtained as a reduct of some ring interpretable in an algebraically closed valued field, and we do not even know its theory. Note that a natural question that arises when studying algebraically closed valued fields is to determine which groups are interpretable in such a structure; our group will appear naturally in that context.

2.

The following definitions can be found in [5] (when we say "definable" we always mean "parameter-definable"):

- A $C$-structure is a structure $(M, C)$ where $C(x ; y, z)$ is a ternary relation satisfying the following axioms:

$-\mathcal{C}_{1}: \forall x y z(C(x ; y, z) \longrightarrow C(x ; z, y))$

$-\mathcal{C}_{2}: \forall x y z(C(x ; y, z) \longrightarrow \neg C(y ; x, z))$;

$-\mathcal{C}_{3}: \forall x y z w[C(x ; y, z) \longrightarrow(C(w ; y, z) \vee C(x ; w, z))]$

$-\mathcal{C}_{4}: \forall x y \exists z[x \neq y \longrightarrow(y \neq z \wedge C(x ; y, z))]$.

- An expansion $\mathbb{M}=(M, C, \ldots)$ of a $C$-structure $(M, C)$ is $C$-minimal if for every elementary extension $\mathbb{M}^{\prime}=\left(M^{\prime}, C, \ldots\right)$ of $\mathbb{M}$, any definable subset of $M^{\prime}$ is quantifier-free definable in $\left(M^{\prime}, C\right)$, that is, by a quantifier-free formula of the language containing only the $C$-relation and the equality.

- A $C$-group is a structure $\mathbb{G}=\left(G, C, \cdot,^{-1}, 1\right)$, where $(G, \cdot,-1,1)$ is a group, $C$ is a $C$-relation and $\mathbb{G}$ satisfies:

$$
\forall x y z u v(C(x ; y, z) \longleftrightarrow C(u x v ; u y v, u z v)) .
$$

A $C$-field is a structure $\mathbb{F}=(F, C,+,-, \cdot, 0,1)$, where $(F,+,-, \cdot, 0,1)$ is a field, and $C$ is a $C$-relation for which both the additive group and the multiplicative group of $\mathbb{F}$ are $C$-groups.

Let $\mathbb{F}=(F,+,-, \cdot, 0,1)$ be a field. From any non-trivial (Krull) valuation $v$ from $F$ to an ordered abelian group, we can define a $C$-relation on $F$ by setting

$$
C(x ; y, z) \text { iff } v(z-y)>v(z-x)
$$

and this makes $(\mathbb{F}, C)=(F,+,-, \cdot, 0,1, C)$ into a $C$-field. Conversely, any $C$-field can be made into a valued field such that the $C$-relation and the valuation satisfy the relation above. It was shown in [5] and [1] that the $C$-minimal $C$-fields correspond to the algebraically closed valued fields. With the induced $C$-relation, the additive group and the multiplicative group of a $C$-minimal $C$-field $\mathbb{F}$ are $C$-minimal groups.

Let $(\mathbb{F}, C)=(F,+,-, \cdot, 0,1, C)$ be an algebraically closed $C$-field and $v$ the corresponding valuation. We use the following notations (for basics on Krull valuations see [6]): $\Gamma$ is the valuation group of $(F, v), A_{v}=\{x \in F \mid v(x) \geqslant 0\}$ the valuation ring and $M_{v}=\{x \in F \mid v(x)>0\}$ its maximal ideal. For any $\gamma \in \Gamma$, $A_{\gamma}=\{x \in F \mid v(x) \geqslant \gamma\}$ and $M_{\gamma}=\{x \in F \mid v(x)>\gamma\}$. We also write $A_{\infty}=\{0\}$ where $\infty$ is the valuation of 0 ( $\infty$ does not belong to the group $\Gamma$ and is greater than any element of $\Gamma$ ). The $C$-field $(\mathbb{F}, C)$ being $C$-minimal, we can easily describe its definable subsets (see [5] for details): any definable subset of any structure elementarily equivalent to $(\mathbb{F}, C)$ is a disjoint union of "truncated cones". A truncated cone in $F$ can be described as a set

$$
D=\left(a_{0}+D_{0}\right) \backslash\left(\left(a_{1}+D_{1}\right) \cup \ldots \cup\left(a_{n}+D_{n}\right)\right)
$$


where $a_{0}, \ldots, a_{n}$ are elements of $F$ and $D_{0}, \ldots, D_{n}$ are equal either to $F$ or to some $A_{\gamma}$, or to some $M_{\gamma}$, where $\gamma \in \Gamma \cup\{\infty\}$. We may assume that $a_{1}+D_{1}, \ldots, a_{n}+D_{n}$ are disjoint subsets of $a_{0}+D_{0}$. We allow the case where $n=0$ and $D=a_{0}+D_{0}$. Remember how these subsets are definable from the $C$-relation: if $v(u)=\gamma$, then $a+A_{\gamma}=\{x \in F \mid \neg C(x ; a+u, a)\}$ and $a+M_{\gamma}=\{x \in F \mid C(a+u ; x, a)\}$.

For any strictly positive $\gamma$, the ring $V_{\gamma}=A_{v} / A_{\gamma}$ can be endowed with the $C$ relation induced by $C$ : for any $x, y, z \in A_{v}, C^{\prime}\left(x+A_{\gamma} ; y+A_{\gamma}, z+A_{\gamma}\right)$ holds if and only if $C(x ; y, z)$ holds and $z-x \notin A_{\gamma}$. Note that the last axiom for $C$-relations holds because the interval $[0, \gamma)$ in $\Gamma$ has no last element since $\Gamma$ is divisible. On the other hand, since $\mathbb{V}_{\gamma}$ is not a domain, the compatibility of the $C$-relation with the product is no longer true. We will call the structure $\left(\mathbb{V}_{\gamma}, C^{\prime}\right)=\left(V_{\gamma},+,-, \cdot, 0,1, C^{\prime}\right)$ a $C$-ring, and denote by $s_{\gamma}$ the canonical morphism from $A_{v}$ to $V_{\gamma}$. Although $C$-minimality is not preserved in general by interpretations, we have

Lemma 2.1. For any strictly positive $\gamma$ the $C$-ring $\left(\mathbb{V}_{\gamma}, C^{\prime}\right)$ is $C$-minimal.

Proof. Every definable subset of $V_{\gamma}$ is the image by $s_{\gamma}$ of a definable subset of $A_{v}$ which is, by $C$-minimality of $(\mathbb{F}, C)$, a disjoint union of truncated cones included in $A_{v}$. Obviously, the parameters used to define these truncated cones can be taken from $A_{v}$. It is easy to see that the image by $s_{\gamma}$ of a truncated cone of $A_{v}$ is a truncated cone of $V_{\gamma}$. We conclude that every definable subset of $\left(\mathbb{V}_{\gamma}, C^{\prime}\right)$ is a disjoint union of truncated cones.

To prove that $\left(\mathbb{V}_{\gamma}, C^{\prime}\right)$ is $C$-minimal we need to verify that every definable subset of every structure elementarily equivalent to $\left(\mathbb{V}_{\gamma}, C^{\prime}\right)$ is a disjoint union of truncated cones. But every such structure $\mathbb{M}$ is an elementary substructure of an ultrapower $\mathbb{N}^{\#}$ of $\left(\mathbb{V}_{\gamma}, C^{\prime}\right)$, and such an ultrapower is interpretable by the same means in an algebraically closed $C$-field. Thus we can apply the preceding argument to $N^{\#}$, and every formula $\phi(\bar{a}, x)$ with parameters in $M$ is equivalent in $N^{\#}$ to a formula $\psi(\bar{b}, x)$ (with parameters in $N$ ) where $\psi(\bar{y}, x)$ is a quantifier-free formula of the language containing only the $C$-relation and the equality. As $\mathbb{M}$ is an elementary substructure $\mathbb{N}^{\#}$, we can find $\bar{c} \in M$ such that $\phi(\bar{a}, x)$ is equivalent in $M$ to $\psi(\bar{c}, x)$.

From now on we assume that $\mathbb{F}$ has characteristic $p>0$.

We define a new operation on $A_{v}$ : let $T$ be an element of $M_{v} \backslash\{0\}$, for any $a, b \in A_{v}$,

$$
a * b=a+b+T a^{p} b .
$$

This operation has the following properties (easy to verify and left to the reader): for $a, b, c \in A_{v}$,

(i) for every positive $\gamma, A_{\gamma}$ and $M_{\gamma}$ are stable under $*$.

(ii) $(a * b) * c=\left(a+b+T a^{p} b\right)+c+T\left(a+b+T a^{p} b\right)^{p} c=a+b+c$ $+T\left(a^{p} b+a^{p} c+b^{p} c\right)+T^{p+1} a^{p^{2}} b^{p} c$ and

$a *(b * c)=a+b+c+T b^{p} c+T a^{p}\left(b+c+T b^{p} c\right)=a+b+c$ $+T\left(a^{p} b+a^{p} c+b^{p} c\right)+T^{2} a^{p} b^{p} c$.

(iii) $a * 0=0 * a=a$.

(iv) $a *\left(-a+T a^{p} a\right)=T^{2} a^{2 p+1}$ and $\left(-a+T a^{p} a\right) * a=T^{p+1} a^{p^{2}+p+1}$.

(v) $\left(\left(-b+T b^{p} b\right) * a\right) * b=a+T\left(a^{p} b-b^{p} a\right)+T^{2} d$, with $d \in A_{v}$.

(vi) $\left(\left(\left(-a+T a^{p} a\right) *\left(-b+T b^{p} b\right)\right) * a\right) * b=T\left(a^{p} b-b^{p} a\right)+T^{2} d$, with $d \in A_{v}$.

(vii) $v(a * c-b * c)=v(c * a-c * b)=v(a-b)$. 
Let $\gamma$ be the valuation of $T$. From the properties above we deduce that $*$ induces on $V_{2 \gamma}$ a group law. By (iv), if $a \in A_{v}$, the inverse of $\bar{a}=a+A_{2 \gamma}$ in $V_{2 \gamma}$ is the element $\bar{a}^{-1}=-a+T a^{p} a+A_{2 \gamma}$. By (vii) this law is compatible with the $C$-relation defined in $V_{2 \gamma}$ : for every $a, b, c, d \in V_{2 \gamma}, \mathbb{V}_{2 \gamma} \models C^{\prime}(a * d ; b * d, c * d)$ if and only if $\mathbb{V}_{2 \gamma} \models C^{\prime}(d * a ; d * b, d * c)$ if and only if $\mathbb{V}_{2 \gamma} \models C^{\prime}(a ; b, c)$. Let $\mathbb{G}=\left(V_{2 \gamma}, *,-1,0, C^{\prime}\right)$ be the $C$-group just defined. Clearly, any $C$-structure that is a reduct of a $C$-minimal structure is again $C$-minimal. As $\mathbb{G}$ is a reduct of $\left(\mathbb{V}_{2 \gamma}, C^{\prime}\right)$, it is a $C$-minimal group.

Consider an element $a \in A_{v}$ and a strictly positive $\gamma \in \Gamma$. Define $Z_{(a, \gamma)}=\{x \in$ $\left.A_{v} \mid v\left(a^{p} x-x^{p} a\right) \geqslant \gamma\right\}$. Its image by $s_{2 \gamma}$ is the centralizer in $\mathbb{G}$ of $a+A_{2 \gamma}$.

Lemma 2.2. (i) if $v(a) \geqslant \gamma$, then $Z_{(a, \gamma)}=A_{v}$,

(ii) if $\frac{\gamma}{p+1} \leqslant v(a)<\gamma$, then $Z_{(a, \gamma)}=A_{\frac{\gamma-v(a)}{p}}$,

(iii) if $0 \leqslant v(a)<\frac{\gamma}{p+1}$, then $Z_{(a, \gamma)}=\bigcup_{n \in \mathbb{F}_{p}}\left(n a+A_{\gamma-p v(a)}\right)$.

Proof. (i) is obvious. Write $x=t a$ with $v(t) \geqslant-v(a)$. Then $x$ belongs to $Z_{(a, \gamma)}$ if and only if $v\left(t-t^{p}\right) \geqslant \gamma-(p+1) v(a)$. If $\gamma-(p+1) v(a) \leqslant 0$ and $v(a)<\gamma$, this means that $p v(t) \geqslant \gamma-(p+1) v(a)$ and $p v(x) \geqslant \gamma-v(a)$ and proves (ii). We now prove (iii): if $\gamma-(p+1) v(a)>0$, then $t$ can be written $t=n+t^{\prime}$ where $n \in \mathbb{F}_{p}$, the field with $p$ elements, and $t^{\prime} \in M_{v}$. Thus $v\left(t^{p}-t\right)=v\left(t^{\prime p}-t^{\prime}\right)=v\left(t^{\prime}\right)$ so $x$ belongs to $Z_{(a, \gamma)}$ if and only if $v\left(t^{\prime}\right) \geqslant \gamma-(p+1) v(a)$.

If $\alpha \in[0,2 \gamma)$, where $\gamma=v(T)$, we call $G_{\alpha}$ the image of $A_{\alpha}$ by $s_{2 \gamma}$. Clearly $G_{\alpha}$ is a subgroup of $\mathbb{G}$. We conclude by:

Theorem 2.3. The group $\mathbb{G}$ is a $C$-minimal group that is not abelian-by-finite. Moreover $\mathbb{G}$ is a nilpotent group of class 2 and of exponent $p$ if $p$ is odd and 4 if $p=2$.

Proof. By the preceding lemma, the set of elements of $V_{2 \gamma}$ whose centralizer is of finite index in $\mathbb{G}$ is equal to $G_{\gamma}$. Since $G_{\gamma}$ is not of finite index in $\mathbb{G}$, the group $\mathbb{G}$ is not abelian-by-finite. It is easy to see that its center is $G_{\gamma}$ and its derived subgroup is also $G_{\gamma}$. Therefore $\mathbb{G}$ is a nilpotent group of class 2 . Finally, computing by induction the $n^{t h}$ power of $a \in A_{v}$, we find the formula $a * a * \ldots * a=$ $n a+T\left(\frac{n(n-1)}{2} a^{p+1}\right)$ modulo $A_{2 \gamma}$.

The valuation $v$ induces a map $v_{2 \gamma}$ from $V_{2 \gamma}$ to the ordered set $[0,2 \gamma) \cup\{\infty\}$ defined by $v_{2 \gamma}\left(a+A_{2 \gamma}\right)=v(a)$ if $v(a)<2 \gamma$, and $v_{2 \gamma}\left(A_{2 \gamma}\right)=\infty$. This map is what we called in [7] a group valuation, and the $C$-group $\mathbb{G}$ belongs to the class of what we called valued $C$-groups: the $C$-relation in $\mathbb{G}$ can be defined from the valuation $v_{2 \gamma}$ by

$$
C^{\prime}(x ; y, z) \text { iff } v_{2 \gamma}\left(z y^{-1}\right)>v_{2 \gamma}\left(z x^{-1}\right) .
$$

In [8] we prove that every $C$-minimal valued $C$-group is nilpotent-by-finite and that every connected (i.e. without proper definable subgroups of finite index) $C$-minimal valued $C$-group of finite exponent is nilpotent. The $C$-group $\mathbb{G}$ defined above is nilpotent of class 2 and we do not have examples of $C$-minimal valued groups of nilpotent class greater than 2 . 


\section{REFERENCES}

[1] D. Haskell, H. D. Macpherson, Cell decomposition of C-minimal structures, Annals of Pure and Applied Logic 66 (1994), 113-162. MR 95d:03059

[2] D. Haskell, E. Hrushovski, H. D. Macpherson, Definable sets in algebraically closed valued fields. Part I: elimination of imaginaries, submitted.

[3] A. A. Ivanov, H. D. Macpherson, Strongly determined types, Annals of Pure and Applied Logic 99 (1999), 197-230. MR 2000j:03050

[4] L. Lipshitz, Z. Robinson, One-dimensional fibers of rigid subanalytic sets, J. Symbolic Logic 63 (1998), 83-88. MR 98m:32049

[5] H. D. Macpherson, C. Steinhorn, On variants of o-minimality, Annals of Pure and Applied Logic 79 (1996), 165-209. MR 97e:03050

[6] P. Ribenboim, The Theory of Classical Valuations, Springer, Berlin 1998. MR 2000d:12007

[7] P. Simonetta, Abelian C-minimal groups, Annals of Pure and Applied Logic 110 (2001), 1-22. MR 2002g:03083

[8] P. Simonetta, On non-abelian C-minimal groups, to appear in Annals of Pure and Applied Logic.

Equipe de Logique Mathématique, Université de Paris ViI, 2, Place Jussieu - case 7012, 75251 PARIS CEDEX 05, France

E-mail address: simbaud@logique.jussieu.fr 